\title{
Lung injury does not aggravate early cerebral inflammation or apoptosis in an animal model.
}

\author{
Kamuf $\mathrm{J}^{1}$, Garcia-Bardon $\mathrm{A}^{1}$, Ziebart $\mathrm{A}^{1}$, Thomas $\mathrm{R}^{1}$, Thal $\mathrm{S}^{1}$, Hartmann EK${ }^{1}$ \\ ${ }^{1}$ Department of Anesthesiology, University Medical Centre of the Johannes Gutenberg-University, Mainz
}

\section{Background and goal of study}

The acute respiratory distress syndrome is not only associated with a high mortality, but also goes along with cognitive impairment in survivors. The cause for this cognitive impairment is still not clear. One possible mechanism could be cerebral inflammation as result of a "lung-brain-crosstalk". Even mechanical ventilation itself can induce cerebral inflammation. We hypothesized, that an acute lung injury aggravates the cerebral inflammation induced by mechanical ventilation itself and leads to neuronal damage.

\section{Materials and Methods}

\section{Subjects: $\quad 20$ pigs (sus scrofa domestica)}

Approval of the state and institutional animal care committee

Groups: Lung injury; central venous injection of oleic acid (OAI, $\mathrm{n}=8$ )

Lung injury; bronchoalveolar lavage

(LAV, $\mathrm{n}=8$ )

Control (Ctr, $\mathrm{n}=6)$

Brain tissue of 4 native animals from a different study served as native group

Methods: Histopathological assessment of hippocampal slices and analysis of mRNA expression of inflammatory key markers by real-time PCR

Statistics: One-way-ANOVA with post-hoc correction for multiple testing. Level of significance $p<0,05$

\section{Results I}

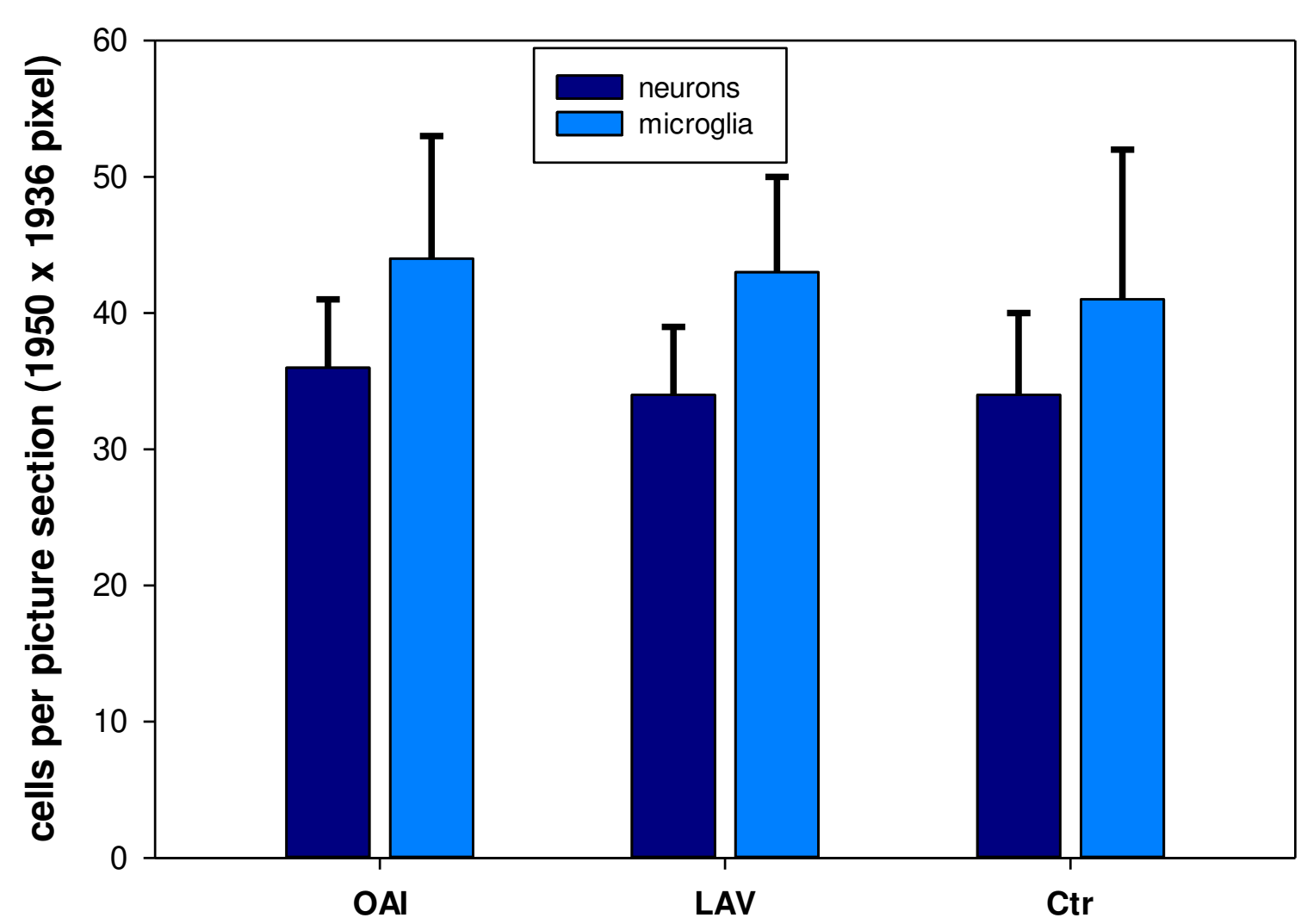

Fig. 1: Histology brain

\section{Results II}

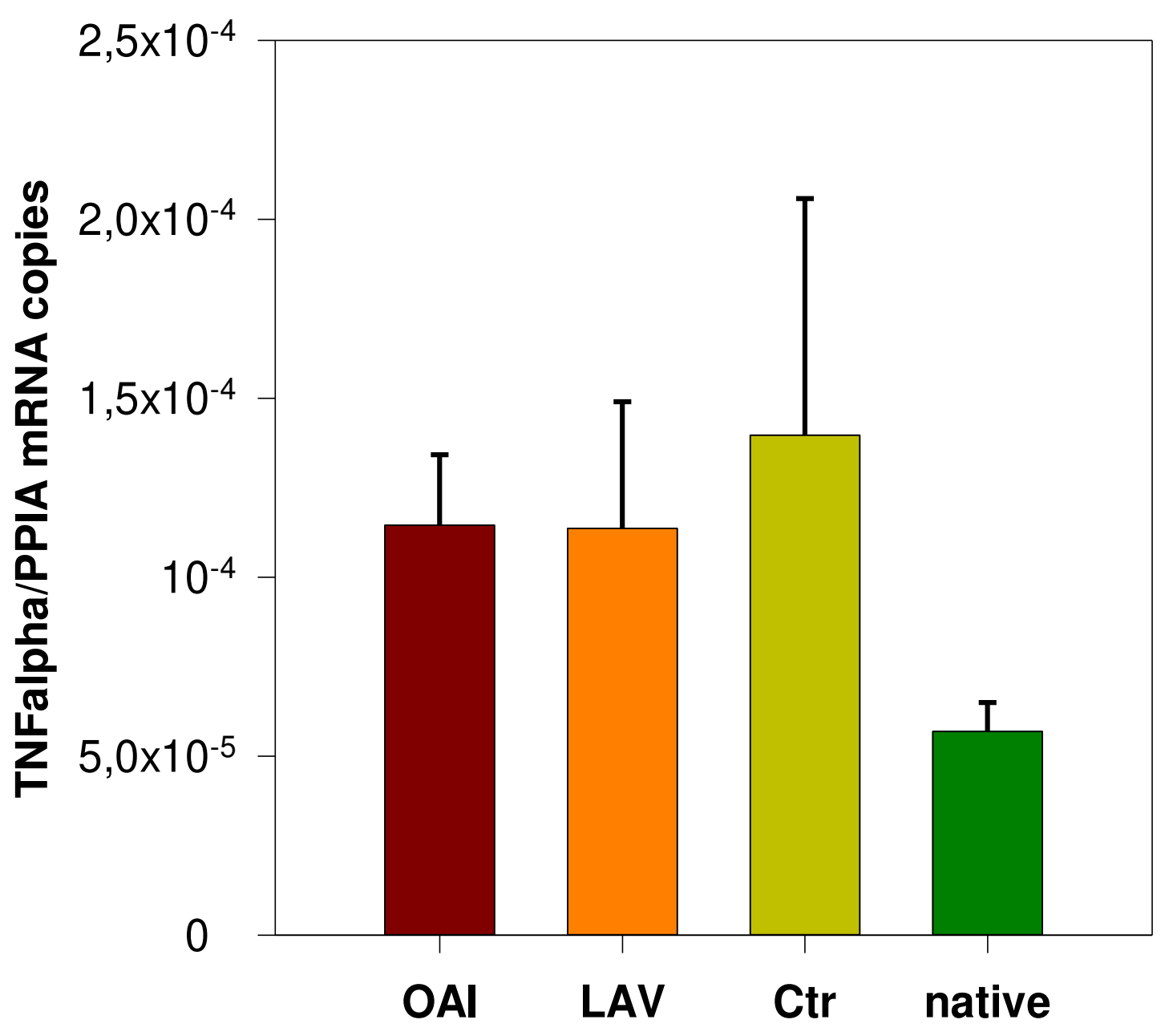

Fig. 2: Expression of TNFalpha in the cortex

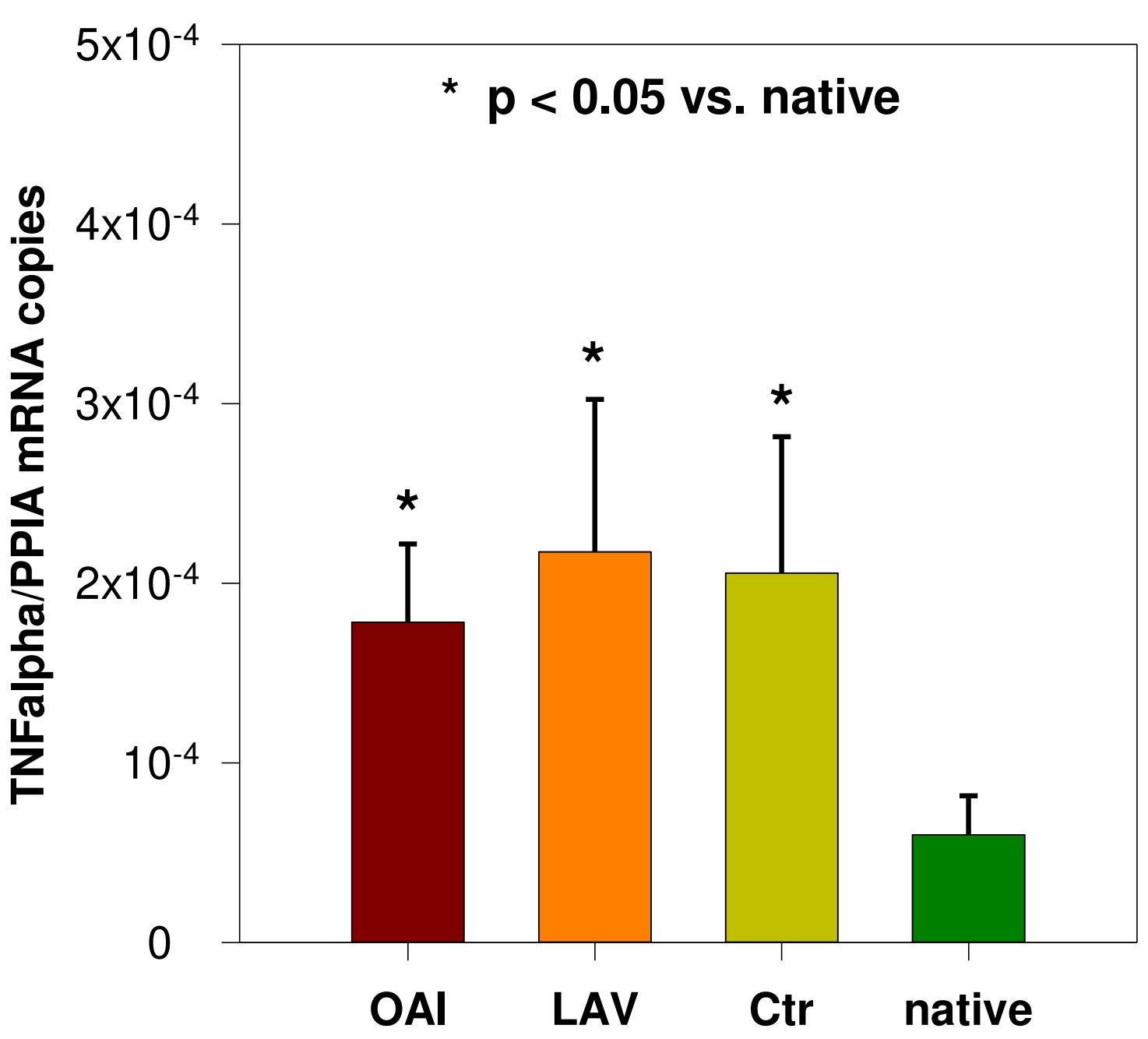

Fig. 3: Expression of TNFalpha in the hippocampus

\section{Conclusions}

With our data we can confirm earlier results, that mechanical ventilation itself seems to trigger cerebral inflammation. This is not aggravated by acute lung injury, at least not within the first 6 hours after onset. Nevertheless, it seems too early to dismiss the idea of lung-injury induced cerebral inflammation, as 6 hours might be just not enough time to see any profound effect 